\title{
Design of Net-learning Systems Based on Experiential Learning
}

\author{
Juan R. Pimentel \\ Department of Electrical and Computer Engineering \\ Kettering University \\ Flint, Michigan 48504 \\ E-mail: jpimente@kettering.edu
}

\begin{abstract}
The theory of experiential learning is briefly reviewed and a model of the learning process is presented. The paper then discusses and characterizes a virtual learning environment and its relationship to experiential learning and learning styles. An approach for designing virtual learning environments is presented taking into account the technology for learning. A prototype for a virtual learning environment designed and built by the author and known as LeProf is then discussed along with its application in the design of an educational site for learning electrical circuits.
\end{abstract}

\section{KEYWORDS}

Experiential Learning, Virtual Learning Environments, Net-learning Systems, Technology for Learning, Object Oriented Environments, Interactive Environments

\section{INTRODUCTION}

Net-learning systems, also known as, Asynchronous Learning Networks (ALNs) are online learning venues that emphasize people-to-people communication combined with traditional and/or information-technology-delivered learning tools [1]. Researchers and practitioners have long been concerned with three fundamental issues involving learning. The first issue involves what people learn--the identifiable knowledge and skills outcomes of learning from accumulated experience. The second issue involves the process of learning (i.e., just how do we learn?), what are the sequences of events and activities that cause or facilitate learning? The third issue is a more practical one and involves a technology for learning (i.e., designing and building learning environments or learning machines to facilitate the learning process). The fundamental idea behind the concept of a technology for learning is a simulated situation designed to create personal experiences for learners that serve to initiate their own process of inquiry and understanding. The objective of this paper is to analyze virtual learning environments and to discuss a method for designing Net-learning systems based on the theory of experiential learning. The paper also discusses a prototype called LeProf along with an application involving the design of a virtual learning environment for a course on electrical circuits.

The paper is structured as follows: Kolb's experiential learning theory is summarized in sections IIA and IIB. Section IIC contains an adaptation of what is known about the process of learning from autonomous intelligent systems to human learning. The state of the art of the technology of learning is discussed in section III. Sections IVA and IVB contain the author's main ideas on how to characterize and design virtual learning environments and more specifically the relationship 
among experiential learning, learning styles and virtual learning environments. To discuss the ideas in a more tangible fashion the author has designed and implemented a prototype known as LeProf, which is discussed in section V. Finally, section VI contain the main conclusions.

\section{THE LEARNING PROCESS}

\section{A. The Experiential Learning Framework}

According to Kolb's experiential learning theory [2], learning is the process whereby knowledge is created through the transformation of experience. In this section we review the theory of experiential learning. Many thinkers have studied the process of learning, most notably Piaget, and much of what we know today comes from their theories. Perhaps the most basic conclusion from these early studies is that people do learn from their experiences particularly from their mistakes. One of the most fundamental requirements that facilitates learning is an appropriate environment where learners can have experiences. Experiential learning emphasizes the role that appropriate environments and experiences play in the learning process. In experiential learning the learner is directly in touch with the realities being studied. It involves direct encounter with the phenomena being studied rather than merely thinking about the encounter or studying the experience of others with such phenomena.

The learning process is not identical for all human beings, and people enter learning situations with a preferred learning style. Associated with this learning style there is a theory about how people learn, or more specifically, about how they themselves learn best. Learning environments that operate according to a learning theory that is dissimilar to a person's preferred style of learning are likely to be rejected or resisted by that person [2]. Thus an understanding of learning environments is important for educational environments based on multimedia and the Internet.

There are two structural dimensions or degrees of freedom that form the basis for any process of experiential learning. As documented by Kolb [2], learning is best facilitated in an environment where there is dialectic tension and conflict between immediate, concrete experience (i.e., reality) and analytic detachment (i.e., abstraction) and this constitutes the first dimension called prehension. In fact, contributions from psychoanalysis provide evidence that the left hemisphere of the human brain is concerned with abstract symbolic representation while the right hemisphere is isomorphic with reality. The second dimension involves the actions of the learner which transforms experience into knowledge and ranges from a totally physically active to a totally passive (i.e., reflective) state and constitutes the transformation dimension. The prehension dimension ranges from concrete experience to abstract conceptualization whereas the transformation dimension ranges from active experimentation to reflective observation resulting in the diagram of Figure 1. This explains why some learners learn by being active (i.e., by having a playful spirit) and trying things out to see what happens. These learners believe in the motto: try it to see if it works. The polarity between concrete experience and abstract conceptualization explains why some learners, young and adult, sometimes favor learning methods which combine work and study, theory and practice resulting in a more familiar and therefore more productive arena for learning. 


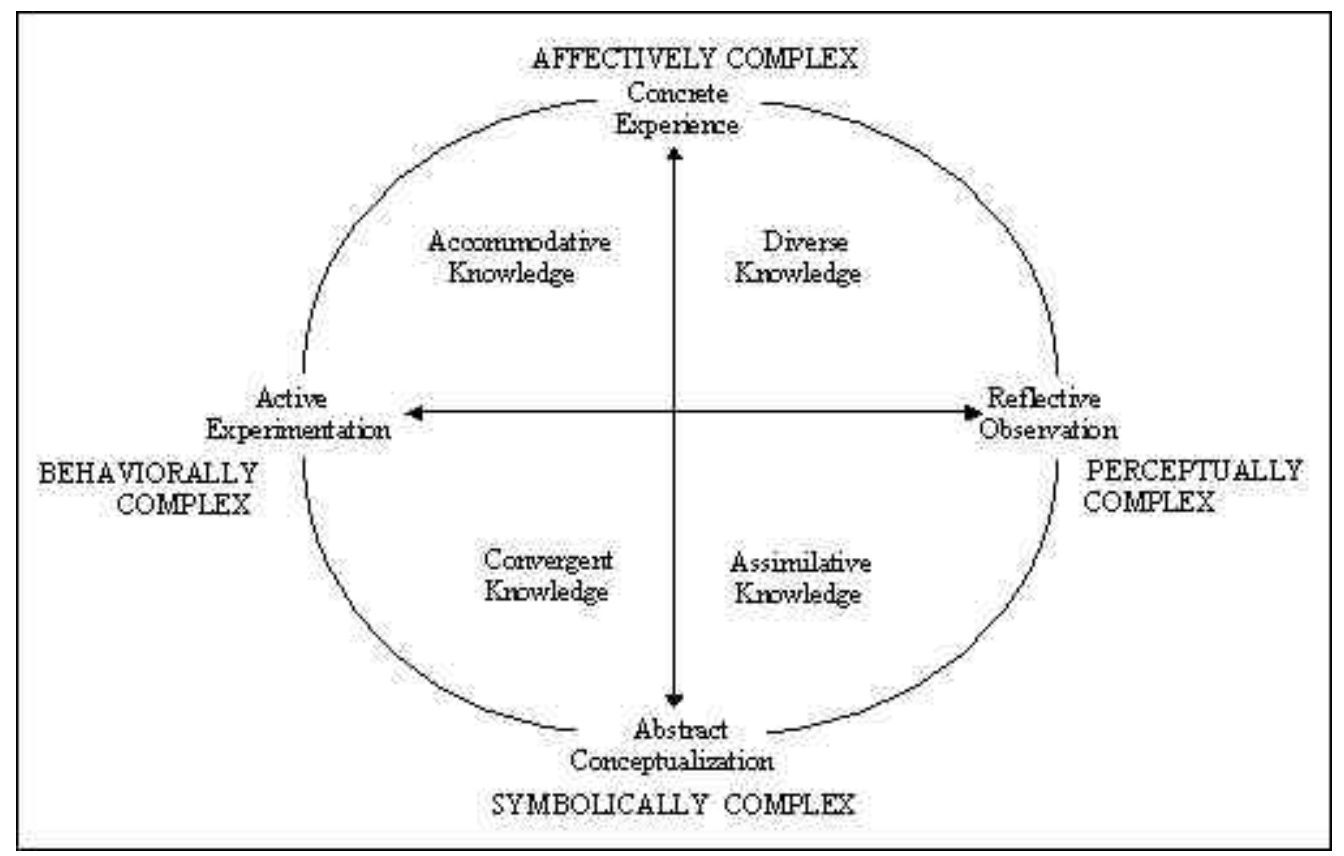

Figure 1. Structural Dimensions Underlying the Process of Experiential Learning and the Resulting Basic Knowledge Forms.

As depicted in Figure 1, the two perpendicular learning dimensions are independent from one another and define four learning modes and four knowledge types. The four learning modes are called concrete experience, reflective observation, abstract conceptualization and active experimentation, and the four knowledge forms are called accommodative, divergent, assimilative and convergent. Individual learning styles can fall in any of the four quadrants defined by the learning modes resulting in four styles called accommodation, divergence, assimilation, and convergence.

As already noted, to effect learning an appropriate environment is required. Learning environments which support the four learning modes depicted in Figure 1 are called respectively affectively complex, perceptually complex, symbolically complex, and behaviorally complex. The following is a summary of these environments [2].

An affectively complex learning environment emphasizes experiencing what it is like to be a professional in the field of study. Learners are engaged in activities that simulate or mirror what they would do as graduates, or they are encouraged to reflect upon an experience to generate these insights and feelings about themselves. The information discussed and generated is more often current and immediate. It often comes from expressions of feelings, values, and opinions by the learner in discussions with peers or the teacher.

The primary goal in a perceptually complex learning environment is to understand something: to be able to identify relationships between concepts, to be able to define problems for investigation, to be able to collect relevant information, to be able to research a question and similar activities. To facilitate this, learners are encouraged to view the topic or subject matter from different perspectives (e.g., their own experience, expert opinion and literature) and in different ways (listen, observe, write, discuss, act out, think, touch and smell). If a task is being done or a problem is being solved, the emphasis is more on how it gets done (i.e., the process) rather than on the solution. 
In a symbolically complex learning environment a learner is involved in trying to solve a problem for which there is a right answer or a best solution. The source of information, topic, or problem being dealt with is abstract, in that it is removed from the present and presented via reading, data, pictures, lecture inputs, and so on. In handling such information, the learner is both guided and constrained by externally imposed rules of inference, such as symbols, computer technology, jargon, theorems, graphical keys, or protocols.

In a behaviorally complex learning environment the emphasis is on the active application of knowledge or skills to a practical problem. For example, the problem need not have a right or best answer, nor does it need to be something the learner can relate to, value or feel some intrinsic satisfaction from having solved. This would normally be a "real-life" problem, case or simulation that the learner could expect to face as a professional. In solving the problem, the focus is on doing.

Learning a scientific subject such as physics or mathematics requires an environment that is primarily symbolically and perceptually complex, whereas learning an engineering subject such as mechanical or electrical engineering requires an environment that is symbolically and behaviorally complex. Likewise, learning the humanities requires an environment that is primarily affectively and perceptually complex, whereas learning management or economic subjects requires an environment that is affectively and behaviorally complex. The appropriate environment for learning a specific subject is not discrete but rather semi-permeable depending upon the learning style of the student.

\section{B. What Do We Learn}

How do we find a proper context appropriate for designing Net-learning systems for all the seemingly huge number of subject areas that one could possibly learn? One approach would be to organize knowledge as done by universities around the world, i.e., the sciences, arts, humanities, medicine, engineering and management. The problem with this approach is that it is not clear how the subject areas are related to how people learn these subjects. Experiential learning provides a framework for organizing what we learn. One of the advantages of the experiential learning framework is that it can also be used for analyzing how people learn and for designing virtual learning environments.

Kolb's experiential learning theory classifies knowledge into one of the following four types: accomodative; divergent; convergent; assimilative, each occupying a quadrant in the concrete/abstract and active/reflective dimensions. Every conceivable subject can be placed precisely in the structural dimensions of experiential learning. For example, Figure 2 depicts the concrete/abstract and active/reflective orientations of several academic fields derived from the Carnegie Commission Study of American Colleges and Universities [2]. It can be noticed that the fields of social work, history, electrical engineering and mathematics have distinct orientations which suggest that learning environments for these fields must vary considerably. 


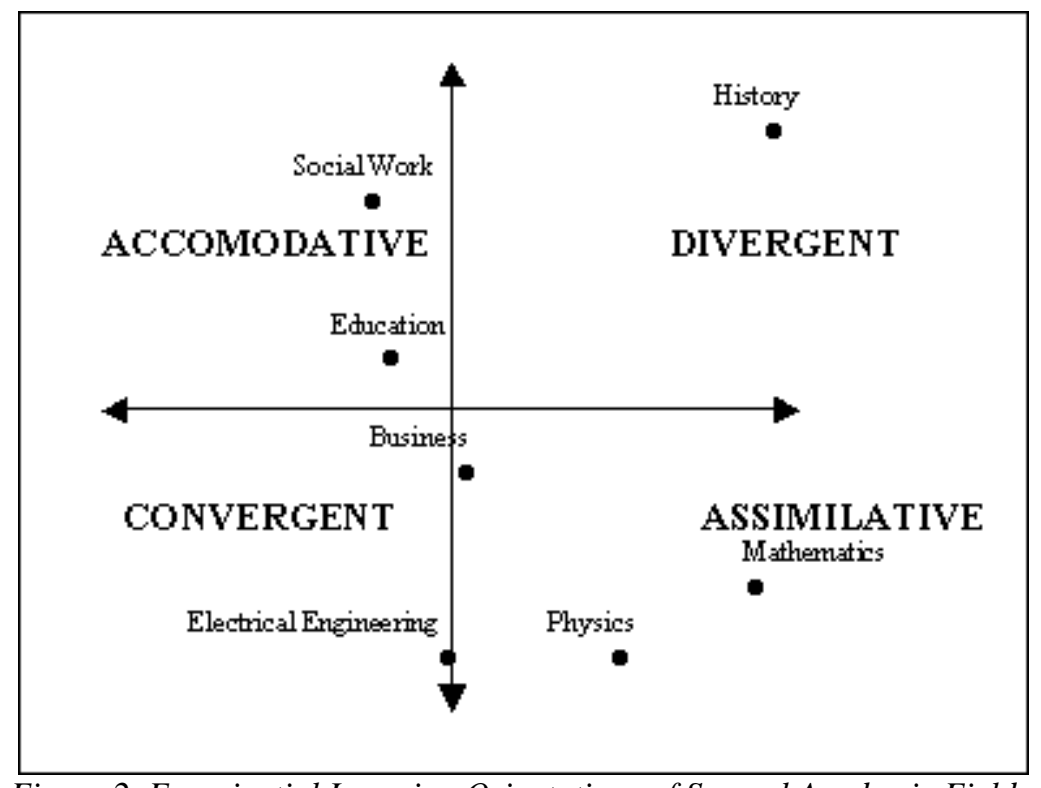

Figure 2. Experiential Learning Orientations of Several Academic Fields.

\section{How Do We Learn}

Knowing how we learn is important because learning environments must take advantage of how learners learn thus optimizing learning. The following is a summary of the process of learning of human beings. The summary has been adapted from other contributions of learning in the context of autonomous intelligent robotic systems [3], [4].

Learning can be viewed as the continuous (e.g., day by day) permanent (i.e., involving memory) incorporation of examples, observations, experiences, situations, rules, concepts and techniques for improving performance in the execution of tasks. At the beginning of the learning process, the knowledge and performance of a learner can be rather low depending upon the initial conditions (e.g., initial knowledge). However, as learners get more experience, it is expected that their performance improve. With sufficient experience, it is expected that the performance of a learner becomes satisfactory in a specific knowledge domain.

Building and storing units of experience in learners can be accomplished through a combination of:

- The human sensory system which can be a combination of various media;

- Initial knowledge or transferred knowledge; (An example of transferred knowledge is the process of reading to learn new things.)

- Building new experiences through an assessment of past experiences.

In addition to building and storing units of experience, learning involves reasoning that enables learners to perform better with more experience. Reasoning also helps the learning process to deal with discovery, understanding and problem solving which have been identified to be closely related to learning [3]. Thus, designing a virtual environment to effect learning is not a trivial matter.

From the foregoing, the following terms are particularly important when discussing learning: mission (involving tasks, subtasks), environment, situation, action, pleasure level, performance 
level, experience and lesson. We next provide a definition of these concepts, adapted and generalized from Fritz, et al. [5], which deals with learning in autonomous mobile robots:

- A sensation, $n$, is the way a learner perceives the virtual environment (i.e., through text, image, animation, simulation and visualization).

- A situation, $s$, is a special condition on the numerical values of a sensation. For example, a particular situation could be an audio explanation of a particular homework problem or assignment.

- An action, $c$, executed by a learner is any activity that transforms the current situation or state into the next situation or state. Actions are performed by the learner on the environment thus changing the situation or state.

- The state, $z$, of the learner is the information necessary to uniquely characterize the learner's progress relative to the execution of a mission.

- The pleasure level, $u$, is the measurement of the pleasure as a result of executing one action or a history of actions.

- The performance level, $P$, of a learner while performing a mission is a set of measures indicating how well the learner is achieving its mission.

- A single experience, $x$, is a tuple of five elements: current situation, action, pleasure level, performance level and next situation.

- A single lesson, $L$, is an abstraction of a set of experiences as it relates to the execution of the mission. A lesson involves learning through experiences. The abstraction could be achieved through generalization or specialization. For example, as a result of the abstraction, a lesson can be represented as a tuple of four elements: action, pleasure level, performance level and task.

- A mission, task, or subtask is to learn a subject, be trained in a specific function or develop competency in a specific endeavor.

\section{LEARNING METHODOLOGIES}

The following summarizes the main learning approaches used by learners. However, before discussing each learning method we provide the following definitions:

- A (learning) rule is a guide for procedures to generate actions based on the state, situation, and performance. Intelligent beings construct rules based on pleasure levels, experiences and lessons.

- An example (of a learning rule) is a record of selected actions to illustrate a model or a set of cases or patterns.

- An advice is an abstraction of a set of rules and examples to cover more general (perhaps unforeseen) situations, performance levels and pleasure levels.

- A solution to a problem is a history of records involving actions, situations, experiences and lessons as it relates to a task including intermediate sub-goals.

All learning approaches to be discussed next have basically the same objective: that of learning the rules to execute a given mission given a set of sensations, examples, advice or solutions to similar problems. Within the context of virtual environments, generating rules typically involves obtaining sensations through human senses, identifying a set of situations and generating the appropriate actions based on the state and performance of the system. In addition, the virtual environment may involve pleasure levels, experience or lessons as defined above. Figure 3 depicts a model for the learning process showing the relationship of the aforementioned concepts. 


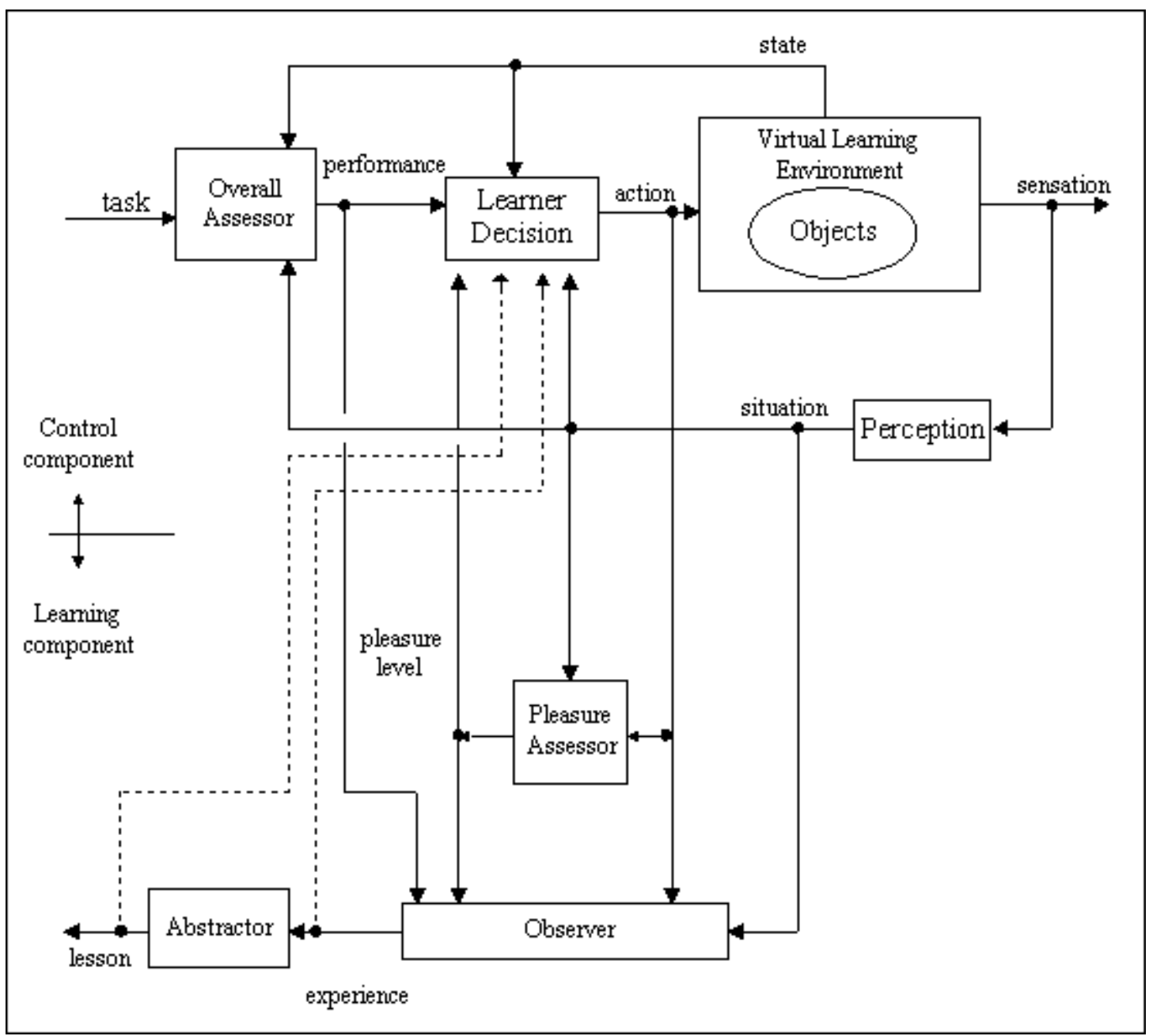

Figure 3. A Model for the Learning Process.

\section{A. Learning Approaches}

\section{Rote Learning}

Rote learning is synonymous with fact memorization and does not involve reasoning, discovery, understanding or problem solving.

\section{Advice Taking}

This is also known as learning from instruction or learning by being told. The learner is required to transform the knowledge of the entity giving advice into an appropriate form to be of effective use and will incrementally augment their knowledge. Learning from taking advice basically involves translating the advice into a set of learning rules. This method parallels most traditional education methods as provided by schools and universities.

\section{Learning From Examples or Evidence}

Given a set of examples and counterexamples of learning actions, the learner induces actions that will hopefully include all of the positive examples and none of the counterexamples. The source generating the examples may include a teacher or the virtual environment. Thus this system generates a set of learning rules given a set of human generated examples. The examples available can include only positive examples or both positive and negative examples. 


\section{Learning by Exploration or Observation}

Nature provides the best example of this paradigm for learning through the generation of some actions and consequent survival of beings. Learning by observation does not include a teacher and consists of a number of processes such as:

- creating classifications of given observations;

- discovering relationships and laws governing a process;

- forming a theory to explain a given phenomenon. [4]

A learning system that is built based on this technique is not provided with any sets of instances or examples of a concept. In addition, the learner may have to deal with a number of observations that represent several concepts rather than just concentrating on a single concept at a time. In the learning approach based on exploration or observation, a learner proceeds in an autonomous fashion for generating its learning rules and appropriate examples. This is the learning approach emphasized in experiential learning and in this paper.

\section{Learning by Analogy}

This involves the solution to a new problem by adapting a known solution to a similar problem. The learner generates a set of rules for solving the new problem.

\section{TECHNOLOGY OF LEARNING}

All the theories of how one learns a specific subject matter are not useful unless virtual learning environments are designed and implemented in a way that learners can start learning. The design and implementation of a virtual learning environment is technology dependent. We live in an exciting period in the history of technology as we are beginning to have key technological developments in place to design truly effective learning environments which could potentially change the way we impart education in fundamental ways and are likely to remain for a long time. With the appropriate technology and tools, it is possible to design virtual learning environments that make use of new methods, paradigms and approaches for teaching and learning.

Because of its nature, the technology for learning environments involves advances in electronics, personal computers, multimedia, the Internet and information technology. Although the technology is not yet mature, the following short discussion involves the most powerful technological elements that significantly affect the quality of learning environments.

\section{A. Multimedia Personal Computers}

Perhaps the most significant development for virtual learning environments is that learners can interact with the environment not with just textual information as in the past, but also using images, voice, video, touch and graphics thus utilizing a media rich interface. Significant advances in the theory of signal, video and image processing, graphics, hardware and processor technology in the past few decades have produced practical and relatively inexpensive devices. These include personal computers, tools for image, voice and video capture, signal processing, rendering, compression and transmission of information over a network. The processing power of multimedia personal computers (PCs) has increased and will continue to increase in the foreseeable future. All of these advances make it possible to design a media rich virtual environment where learners, for example, can interact with a simulated view of a process, 
mechanism or device being studied. Most Net-learning systems are required to run on multimedia PCs.

\section{B. Internet and Intranets}

Although the Internet was developed in the late 60's, its widespread use did not occur until 1994 when the first Web browser Mosaic was made public at the University of Illinois. The significance of the Internet for Net-learning systems is that it allows users to geographically transcend the boundaries of a campus, institution, city, state, province or country. Content can be accessed and available to anyone where there is an Internet connection thus providing a learn anywhere feature. Although some components (e.g., videoconferencing) can be accessed synchronously, the Internet also allows learners to access the virtual environment at any time, i.e., asynchronously. To provide the learn anywhere, anytime feature, currently all Net-learning systems are required to be Internet based.

Transmitting content of virtual environments places stringent requirements on the network (Intranet or Intranet) and more specifically voice and video. Two critical Internet issues affect the performance of virtual learning environments: communication devices and protocols. Latest advances in communication devices such as asynchronous transfer mode (ATM) with speeds of 155 to 622 million bits per second, 100 million bit per second Ethernet and Gigabit Ethernet (one billion bits per second) will help improve performance. Unfortunately, most Internet protocols in use today were not designed to transmit real-time information which is useful for synchronous content and for transmitting rich content. More appropriate protocols (e.g., multicast protocols) are being developed by the Internet Engineering Task Force (IETF) and they are expected to be widely used in the next few years. Although the Internet is made up of many protocols, Table 1 shows the protocols that are significant for designing and implementing virtual learning environments. Other emerging Internet protocols that are relevant for virtual environments are discussed briefly in section IIIK.

\begin{tabular}{|l|l|l|}
\hline Supported Application & $\begin{array}{l}\text { Protocol } \\
\text { Acronym }\end{array}$ & Protocol Name \\
\hline Sending/Receiving mail & SMTP & Simple mail transfer protocol \\
\hline File manipulation & FTP & File transfer protocol \\
\hline Remote computer access & TELNET & Virtual terminal network protocol \\
\hline Sending/Receiving web pages & HTTP & Hypertext transfer protocol \\
\hline $\begin{array}{l}\text { Sending/Receiving raw data } \\
\text { unreliably }\end{array}$ & UDP & User Datagram Protocol \\
\hline $\begin{array}{l}\text { Sending/Receiving raw data } \\
\text { reliably }\end{array}$ & TCP & Transmission Control Protocol \\
\hline
\end{tabular}

Table 1. Internet Protocols Useful for Designing Virtual Learning Environments.

\section{Client-server Systems}

A client-server system is the most used method of organizing software within computers connected to the Internet. A client-server system is basically a system where components called servers have resources and information that other components called clients wish to access. Clients connect to a server to obtain the desired resources or information and when the information is obtained they disconnect, like using a telephone. The significance of client-server systems is that they work with the Internet in a synergistic fashion thus allowing clients and servers to be geographically dispersed. Many of the elements of Net-learning systems are best designed using the client-server paradigm. Client-server systems have become so pervasive that 
much of the current Internet technology is classified as client technology or server technology. Client technology tools are significant for learners because they are the tools that learners are expected to acquire, buy and use. Server technology tools are important for designers and institutions providing the virtual environment as they support the infrastructure needed to effect Net-learning systems.

\section{World Wide Web}

The development of the World Wide Web (WWW) has allowed the Internet to be useful for all society sectors rather than just scientists, engineers and professors, which was the case before its introduction in 1994. The Web is simply a way to access resources using the uniform resource locator (URL) located anywhere on the Internet with the use of hyperlinks and a user-friendly graphical user interface called a Web browser. The two major components of the WWW are hypertext transfer protocol (HTTP), the protocol used to access the resources, and hypertext markup language (HTML), a language to encode hyperlinks and other content.

The WWW has made possible the distribution and publishing of online educational materials. The WWW is significant for the design of Net-learning systems because HTML acts as the glue that holds the elements of the system together and links related information. Although a simple and straightforward language, HTML allows the use of more complex tools (e.g., Java applets and other software plug-ins) to handle more sophisticated and complex functions (e.g., visualization, animation and simulation).

\section{E. Computer Based Training}

Computer based training (CBT) consists of sophisticated educational and training systems that predate the Web and have been useful for developing content with a high degree of simulation, animation, visualization and interaction. CBT systems are important in that much of their functionality is desired in Net-learning systems, and virtual learning environments are just beginning to provide this functionality in an integrated fashion. Much can be learned from CBT systems and the challenge is to integrate their functionality in virtual learning environments.

\section{F. Conferencing}

Conferencing is an asynchronous tool that had its beginnings with network news groups based on the network news transfer protocol (NNTP). Virtual learning environments require that conferencing systems be Web based, allow several threads of discussion and that content be organized by date and subject.

\section{G. Audio and Video}

Some content could benefit immensely if audio and video were added to textual information. The technology of audio and video for virtual learning environments is totally digital, very different from that used in traditional broadcast radio and television. There are basically two ways that one can render audio and video in a PC: streaming or playback. Streaming data sources include broadcast media, multicast media and video-on-demand (VOD). Playback involves playing the audio or video from local files in a way similar to playing a VCR. Incorporating audio and video content in virtual learning environments is challenging because current bandwidth limitations imposed by communication devices make streaming video impractical and the requirement to download and install proprietary browser plug-ins (and their update) to receive audio and video is not easy or desirable for most learners. 


\section{H. Object Oriented Software}

Object oriented systems constitute one of the most powerful and recent paradigms of complex software design and implementation. The main advantage of an object oriented system in the design and implementation of educational software is that it reduces the details first orientation that can cause designers to get so immersed in details that it becomes extremely difficult to develop adequate larger modules of instruction. Using an object-oriented methodology, the modules developed should be easily reusable or modifiable [6], [7]. There are several objectoriented environments and languages such as Smalltalk, C++, Visual Basic and Java. All of the elements of Net-learning systems can be designed and implemented using the object oriented paradigm and software.

\section{User Interface}

The user interface is important because it is the way learners interact with the virtual learning environment. Traditional graphical interface technologies are $\mathrm{X}$-windows and Microsoft windows. Java also provides a more powerful and user-friendly graphical interface at a higher level, which translates into X-windows or Microsoft windows depending upon the platform.

\section{J. Java}

Java is a powerful programming language and object development framework developed by Sun Microsystems for integrating multimedia elements in an Internet environment [8]. One of Java's main advantages is that it provides the capability to build systems and components which are platform independent (i.e., open), object-based and modular. Java simplifies the design and implementation of systems which are scalable and based on reusable components. It allows a high level of interactivity and has a graphical user interface (GUI) to support simulation, animation and visualization. Sun continues to add application program interface (API) to the language. One of the newest additions is APIs for audio and video, 2D and speech. The importance of Java for virtual learning environments is that it provides an appropriate technology for adding multimedia functionality to web content and allows for the creation of more sophisticated user interfaces within a browser.

\section{K. Emergent Technologies and Standards}

The main emerging technologies that will impact virtual learning environments involve communication devices/infrastructure and more appropriate protocols to support multimedia communications. Regarding the communication devices/infrastructure, in addition to Asynchronous Transfer Mode (ATM) and 100 Mbps and Gigabit Ethernet, the development and deployment of Asynchronous Digital Subscriber Line (ADSL) and cable modem technology will be important [9]. These technologies will provide the necessary bandwidth to transport multimedia traffic to residential areas. Regarding more appropriate protocols to support multimedia communications, the traditional Internet protocols (see Table 1) were designed for applications that require high reliability but not real-time operation that is required when voice and video are sent over the network. For applications requiring streaming voice and video an emergent protocol known as real time protocol (RTP) and its companion real time control protocol (RTCP) is gaining popularity [10]. It is expected that the next-generation of WWW browsers will also use RTP for live video and audio streams [11]. These new protocols are called multimedia protocols and are designed to handle a variety of media types and encoding schemes (e.g., H.263, H.323, MPEG and JPEG).

Another important emerging technology involves multicast protocols which are crucial for group communications and collaboration. Once all key protocols and infrastructure are in place, it will be easy to design and implement videoconferencing component tools which could be easily 
integrated within virtual learning environments. Table 2 depicts the network architectures of the traditional Internet and the emerging Internet supporting audio and video streams.

\section{Technology Integration}

A Net-learning system is an interconnected system of learning tools that is constructed using various technologies. As a result it is necessary to integrate these tools using an appropriate software and development environment.

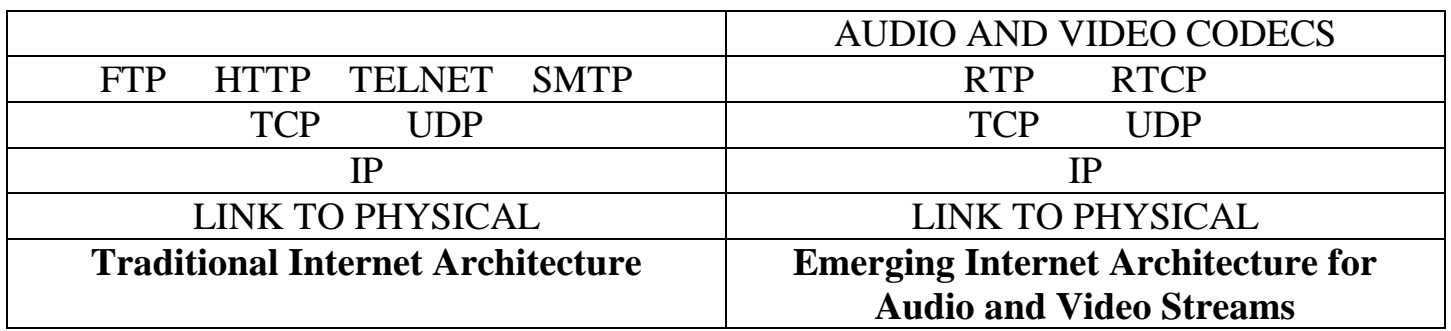

Table 2. Network Architectures of Traditional and Emerging Internet.

\section{VIRTUAL LEARNING ENVIRONMENTS}

Up to this point I have been summarizing Kolb's experiential learning theory, adapting what is known about the process of learning from autonomous intelligent systems to human learning, and discussing the state of the art of the technology of learning. The remainder of the paper contains the author's main ideas on how to characterize and design virtual learning environments and more specifically the relationship among experiential learning, learning styles and virtual learning environments. To discuss the ideas in a more tangible fashion the author has designed and implemented a prototype known as LeProf, which is discussed in section V.

The challenge to the characterization and design of Net-learning systems is that they must provide an effective learning environment. Although most educators agree that to improve the learning process we must have appropriate learning environments, there is not yet a universal definition of a learning environment and more specifically of a virtual one. Perhaps schools, universities, and other educational institutions are the best learning environments that have been invented so far. For some subjects, all that is needed to constitute a learning environment is some buildings, classrooms, faculty offices, faculty with whom students can interact, a library and fellow classmates. For other subjects (e.g., engineering) this is not enough. There must be laboratories, adequate equipment and components, and a well-designed set of laboratory experiments. Engineering students will learn more from a set of laboratory experiences than from simply reading a book or talking to others who have read the book.

What constitutes a virtual learning environment for one educator may not be such for another. Educators, whose area of expertise involves a knowledge type, tend to view a virtual learning environment as one supporting just that knowledge type. For the purposes of this article, we define a virtual learning environment as one that allows learners to perceive the environment, assess situations and performance, perform actions and proceed through experiences and lessons that will allow them to perform better with more experience on repetition of the same task in similar circumstances. This definition of a virtual learning environment emphasizes the importance of learning. Learners in a virtual environment are expected to make use of and include examples, observations, experiences, situations, rules, concepts and techniques in a 
continuous (e.g., day by day or week by week), permanent (i.e., committing knowledge into memory) fashion to improve the performance of the execution of tasks.

Designing effective virtual learning environments is not a trivial task. As Bourne has pointed out in [1], there are two challenges: 1) How to design an environment to enable students to learn better and 2) how to design an environment that will enable learning outside the classroom and provide improved learning experiences for the students. Experiential learning more precisely enables the characterization of a virtual learning environment and helps in designing virtual learning environments to meet the challenges outlined above. More specifically, we can identify the main features of the four learning environments defined by experiential learning and use these features to design virtual learning environments. Table 3 lists the features of each of the four learning environments.

\begin{tabular}{|c|c|c|c|c|}
\hline Environment Feature & Affectively & Perceptually & Symbolically & Behaviorally \\
\hline $\begin{array}{l}\text { Lecture notes } \\
\text { Slides, text } \\
\text { Slides, text with audio } \\
\text { Slides, text with audio and } \\
\text { video }\end{array}$ & $\checkmark$ & $\sqrt{ }$ & $\checkmark$ & $\sqrt{ }$ \\
\hline Theory readings & & $\sqrt{ }$ & $\checkmark$ & \\
\hline Case studies & $\sqrt{ }$ & & & $\sqrt{ }$ \\
\hline Exercises, homework, quizzes & & $\checkmark$ & $\checkmark$ & \\
\hline Visualization & & $\checkmark$ & $\sqrt{ }$ & \\
\hline Animation & & $\checkmark$ & $\sqrt{ }$ & \\
\hline Simulation & & $\sqrt{ }$ & $\sqrt{ }$ & \\
\hline Peer feedback & & & & $\checkmark$ \\
\hline Personalized feedback & & & & $\checkmark$ \\
\hline Feelings are shared & $\sqrt{ }$ & & & \\
\hline $\begin{array}{l}\text { Apply skill/solve problem } \\
\text { activity }\end{array}$ & & & & $\sqrt{ }$ \\
\hline Teacher is coach/helper & & & & $\checkmark$ \\
\hline Teacher is expert/interpreter & & & & $\sqrt{ }$ \\
\hline Teacher as task master/guide & & $\sqrt{ }$ & $\checkmark$ & \\
\hline Teacher as model of profession & $\checkmark$ & & & \\
\hline Expert talk/seminar & $\checkmark$ & & & \\
\hline $\begin{array}{l}\text { Self directed (autonomous) } \\
\text { learner }\end{array}$ & & $\sqrt{ }$ & $\sqrt{ }$ & \\
\hline Learner thinks alone & & $\sqrt{ }$ & & \\
\hline $\begin{array}{l}\text { Learner experiences being a } \\
\text { professional }\end{array}$ & $\sqrt{ }$ & & & \\
\hline $\begin{array}{l}\text { Learner determines own criteria } \\
\text { of relevance }\end{array}$ & & & $\sqrt{ }$ & \\
\hline Focus on process & & $\checkmark ?$ & & \\
\hline $\begin{array}{l}\text { Information focus on tasks and } \\
\text { their completion }\end{array}$ & & $\checkmark$ & $\sqrt{ }$ & \\
\hline Symbolic Tool & & & $\checkmark ?$ & \\
\hline $\begin{array}{l}\text { Information source is here and } \\
\text { now }\end{array}$ & & & & $\checkmark$ \\
\hline
\end{tabular}




\begin{tabular}{|l|c|l|l|l|}
\hline Small group discussion & $\checkmark ?$ & & & \\
\hline Conferencing & & & & \\
\hline Synchronous broadcast & & & & \\
\hline $\begin{array}{l}\text { Performance judged right or } \\
\text { wrong }\end{array}$ & & & $\checkmark$ & \\
\hline $\begin{array}{l}\text { Step by step solution to } \\
\text { problem }\end{array}$ & & & & $\checkmark ?$ \\
\hline
\end{tabular}

? Indicates a fundamental feature

$\checkmark$ Indicates an auxiliary feature

Table 3. Environmental Features of the Learning Environments Defined by Experiential Learning.

The features of the environment, the situations, actions, experiences and lessons differ depending upon the subject matter. What is appropriate for one environment may not be appropriate for another environment. For example, for graduate M.B.A. architecture students the learning environment feature of providing personalized feedback has a correlation coefficient of 0.45 (helpful) when the learning style is concrete experience and a correlation coefficient of -0.47 (not helpful) when the learning style is abstract conceptualization [2].

\section{A. Learning in Virtual Environments}

To optimize learning, learners must perform the following activities:

- Perceive the environment

- Assess situations, state and performance

- Perform actions

- Link actions, situations, state and performance

- Assemble experiences and lessons

Learners perceive the virtual environment through the multi-sensory nature of a multimedia client computer, i.e., through images, text, graphics, sound and video in an interactive fashion. The perception of the environment is enhanced using animation, visualization and simulation. Once learners perceive an environment they are in a position to assess various situations and the learner state. In the field of electrical circuits, a situation could be characterized in terms of values for voltages and currents in certain circuit elements. The state can be the learner's knowledge, relative progress, and test scores on a specific course topic. Actions involve activities such as accessing a conference system, posting a message, working a homework problem and choosing to visualize the solution to a problem. Linking actions, situations, state and performance requires that the learner make a concerted effort involving understanding, discovery and problem solving. The final step involves assembling and organizing experiences and lessons for future reference.

The challenge is to design appropriate environments with rich experiences to effect learning. Actions that involve hands-on activities, performed by the learner, require that the environment provide a high level of interactivity. The lessons are conclusions formed in the mind of learners associating experiences, actions and performance. One can think of a lesson as the correlation that the human mind makes relating an action to a task and how well the action contributes to the completion of a task. Thus, the environment does not provide a lesson in an explicit fashion, rather it is something that humans do naturally. The environment simply provides the means for lessons to be learned. Once a learner goes through all the activities aforementioned in the order indicated it will result in the creation of knowledge, i.e., learning is effected. Table 4 lists some examples of situations, states, pleasure levels, performance levels and actions in a virtual learning environment. 


\begin{tabular}{|c|c|}
\hline Situations & $\begin{array}{l}\text { Audio explanation of a problem } \\
\text { Visualization of solution to a problem } \\
\text { Receiving various degrees of help and } \\
\text { feedback } \\
\text { Being encouraged to think and reason } \\
\text { A high resolution image } \\
\text { Viewing answers to questions or problems } \\
\text { Being close to completing a course } \\
\text { A videoconferencing session }\end{array}$ \\
\hline States & $\begin{array}{l}\text { Learner is behind homework schedule } \\
\text { Learner is deficient in some of content } \\
\text { background } \\
\text { Learner understands concepts but has } \\
\text { difficulty } \\
\text { applying them in applications. }\end{array}$ \\
\hline Pleasure levels & $\begin{array}{l}\text { Discomfort in repeatedly making mistakes } \\
\text { Discomfort while submitting incorrect } \\
\text { answers } \\
\text { Discomfort as a result of being clueless on } \\
\text { solving Problems } \\
\text { Happiness in gaining insight through } \\
\text { multimedia } \\
\text { Happiness on submitting a correct answer to } \\
\text { a } \\
\text { question or problem } \\
\text { Happiness on fast progress }\end{array}$ \\
\hline Performance & $\begin{array}{l}\text { Grades on homework, tests and exams } \\
\text { Submission of work on time } \\
\text { Learning progress made to date } \\
\text { Percent of course completed to date } \\
\text { Degree of participation in discussion forums }\end{array}$ \\
\hline Actions & $\begin{array}{l}\text { Submit input to a discussion forum } \\
\text { Input answer to questions. } \\
\text { Request various degrees of help. } \\
\text { Request suggestions } \\
\text { Choose to visualize concepts, answers } \\
\text { Choose to perform a simulation } \\
\text { Probe cause-effect relationships through } \\
\text { simulation } \\
\text { Request to view grades and other } \\
\text { performance } \\
\text { Interactively solve a problem step by step }\end{array}$ \\
\hline
\end{tabular}

Table 4. A Sample of Situations, States, Pleasure Levels, Performance Levels and Actions. 


\section{B. Design of Net-learning Systems}

The design of Net-learning systems must take into account the various learning environments summarized in Table 3 (i.e., what people learn), appropriate experiences that can be constructed from situations, states, pleasure levels, performance levels actions as listed in Table 4 (i.e., how people learn), and the technology of learning as summarized in section III. Given a subject matter to be learned, designing a Net-learning System involves developing appropriate learning experiences and a virtual environment to provide such experiences making use of appropriate technology.

There is not yet any universal definition identifying the component elements of a Net-learning system [1]. J. Bourne has divided the major elements of Net-learning into 50\% self-learning (CBT and online materials) and 50\% learning with others (conferencing and synchronous communication). The objective of this section is to identify the main components of Net-learning systems and to discuss main design approaches.

From Table 3, we distinguish fundamental features (marked with ?) and several auxiliary features (marked with $\checkmark$ ) per learning environment. Tables 5 through 8 list only the most relevant (fundamental and auxiliary) features in each learning environment.

\begin{tabular}{|l|l|}
\hline Fundamental & Small group discussion \\
\hline Auxiliary & Lecture notes \\
& Slides, text \\
& Slides, text with audio \\
& Slides, text with audio and video \\
& Case studies \\
& Feelings are shared \\
& Teacher as model of profession \\
& Expert talk/seminar \\
& Learner experiences being a professional \\
& Conferencing \\
& Synchronous broadcast \\
\hline
\end{tabular}

Table 5. Affectively Complex Learning Environment Features.

\begin{tabular}{|l|l|}
\hline Fundamental & Focus on process \\
\hline Auxiliary & Lecture notes \\
& Slides, text \\
& Slides, text with audio \\
& Slides, text with audio and video \\
& Theory readings \\
& Exercises, homework, quizzes \\
& Visualization \\
& Animation \\
& Simulation \\
& Teacher as task master/guide \\
& Self directed (autonomous) learner \\
& Learner thinks alone \\
& Information focus on tasks and their \\
& completion \\
\hline
\end{tabular}

Table 6. Perceptually Complex Learning Environment Features. 


\begin{tabular}{|l|l|}
\hline Fundamental & Symbolic Tool \\
\hline Auxiliary & Lecture notes \\
& Slides, text \\
& Slides, text with audio \\
& Slides, text with audio and video \\
& Theory readings \\
& Exercises, homework, quizzes \\
& Visualization \\
Animation & Simulation \\
& Teacher as task master/guide \\
& Self directed (autonomous) learner \\
& Learner determines own criteria of \\
& relevance \\
& Information focus on tasks and their \\
& completion \\
Performance judged right or wrong \\
\hline
\end{tabular}

Table 7. Symbolically Complex Learning Environment Features.

\begin{tabular}{|l|l|}
\hline Fundamental & Step by step solution to problem \\
\hline Auxiliary & Lecture notes \\
& Slides, text \\
& Slides, text with audio \\
& Slides, text with audio and video \\
& Case studies \\
& Peer feedback \\
& Personalized feedback \\
& Apply skill/solve problem activity \\
& Teacher is coach/helper \\
& Teacher is expert/interpreter \\
& Information source is here and now \\
\hline
\end{tabular}

Table 8. Behaviorally Complex Learning Environment Features.

Based on the previous tables we distinguish four virtual learning environments each having a fundamental feature and several auxiliary features as listed in Table 9. The features will be supported by appropriate modules. 


\begin{tabular}{|l|l|l|}
\hline Learning Environment & Fundamental Module & Auxiliary Modules \\
\hline Affectively Complex & Collaborative & $\begin{array}{l}\text { Course content } \\
\text { Media Library } \\
\text { Synchronous }\end{array}$ \\
\hline Perceptually Complex & Process & $\begin{array}{l}\text { Course content } \\
\text { Media Library } \\
\text { Assessment } \\
\text { Course navigation }\end{array}$ \\
\hline Symbolically Complex & Symbolic & $\begin{array}{l}\text { Course content } \\
\text { Assessment } \\
\text { Course navigation }\end{array}$ \\
\hline Behaviorally Complex & Guidance & $\begin{array}{l}\text { Course content } \\
\text { Media Library } \\
\text { Collaborative } \\
\text { Applications }\end{array}$ \\
\hline
\end{tabular}

Table 9. Fundamental and Auxiliary Modules and Virtual Learning Environments.

From the previous tables we identify the following fundamental modules: Collaborative, Process, Symbolic and Guidance and the following auxiliary modules: Course content, Media Library, Synchronous (Video Conferencing), Assessment, Course navigation (e.g., schedule and homework) and Applications (where learners apply their skills or solve problems). The virtual learning community has identified the collaborative fundamental module as crucial and many commercial and non-commercial environments support this feature. There are several collaborative component tools available that could be easily integrated with other tools to form a more complete environment. The remaining fundamental modules (Process, Symbolic and Guidance) have not been explicitly recognized. Designing and implementing these three modules are not trivial, as they are complex and highly dependent on content. The author is not aware of any commercial examples that can be cited for these three modules.

A Process Module focuses on the approach taken while analyzing a subject or solving a problem and has to be highly interactive with rich content. The Symbolic Module provides context, formalism, tools and mechanisms to deal with highly technical issues. This module has similar functionality of tools such as Mathcad, Matlab, Maple, Mathematica, and Saber which are highly specialized analysis, simulation and design tools for electrical and mechanical engineering subjects. However one solution that is beginning to be used to provide the functionality of the Symbolic Module is to provide links to the aforementioned tools (e.g., to Mathematica) and in this way to integrate the tool with the virtual learning environment. Progress in this area will benefit from emerging standards such as Mathematical Markup Language (MML). Conceptually, the Guidance Module is less complex but needs to provide many details while guiding a learner on a project or how to solve problems. Another difficulty in designing this module is that to be useful it has to include a large number of cases to cover a subject with enough depth and breadth.

To support a rich set of experiences, virtual environments must also support the following features: total immersion, interactivity, animation, visualization, simulation, immediate response and information rich (to allow learners to determine their own criteria of relevance). Thus content of virtual learning environments must also include modules supporting the aforementioned features.

In addition to providing all the modules in one of the four learning environments (depending on the subject matter), a virtual learning environment should provide some modules in the remaining 
three learning environments. The reason for this is based on Kolb's experiential learning theory where learners may have learning styles that could benefit from modules in the other three environments. Based on the decision of whether to build a virtual environment from its constituent components or use an already developed virtual environment, we have the following two approaches for designing a Net-learning System:

\section{Design Based on Using an Already Developed Virtual Environment}

This approach involves acquiring and using a turn-key system having most or all of the desired features in a given learning environment and is suitable to educators who do not have time, interest or knowledge to deal with individual constituent components. The modules and tools offered in the virtual environment are fixed and the designer has to create appropriate content and learning experiences. The advantage of this design approach is that it is relatively simple as the designer concentrates on content and experiences and not on integration of components or designing new components from scratch. However the disadvantage is that there is no turn-key system comprehensive enough to support the four learning environments. An example of an integrated tool to support an affectively complex learning environment is LearningSpace ${ }^{\mathrm{TM}}$, of the Lotus Development Corporation, which is highly effective because it is based on a collaborative learning tool which is a fundamental module for this learning environment.

\section{Design Based on Building a Virtual Environment From its Constituent Components}

This approach involves integrating third party and locally developed components into a single system. Depending upon the number of components used and degree of integration required, this approach requires some knowledge and experience on the part of the designer(s). The motivation for using this approach is that the technology is not mature enough and thus the designer has considerable freedom to use novel ways to perform the integration or to design brand new components from scratch. The main advantage of this approach is that it is possible to design learning environments that effectively support any learning environment with advanced features to improve learning.

\section{LEPROF}

LeProf is a virtual learning environment that has been designed based on building the environment from its constituent components. Some components are commercial while others are locally developed because of their unavailability as third party tools. LeProf primarily supports a symbolically complex learning environment but it also includes modules from other experiential learning environments to enhance student learning with various types of learning styles. Thus, LeProf can be used to teach subject content in the category that belongs to the symbolically complex environment such as engineering and physics. More modules need to be developed to enable LeProf to support the remaining three learning environments.

To enable students to learn better and to provide improved learning experiences, an appropriate set of experiences needs to be designed and incorporated into the virtual environment. This step requires that the actual subject matter be specified and this constitutes the application context. The initial version of LeProf supports an introductory course on electrical circuits for non-majors. Once the application context is defined, one can design a comprehensive list of situations, states, pleasure levels, performance and actions such as those listed in Table 4. To enable learning outside the classroom, the learning environment has to be self-contained, meaning that all modules and tools need to be comprehensive, complete, and contain a high level of detail to 
ensure that students can successfully navigate and complete their course work mostly on their own. The initial content on electrical circuits is intended to support an on-campus course but other content could be developed to support learning outside the classroom (off-campus version). Table 10 lists the modules currently implemented by LeProf along with corresponding tools and other components.

\begin{tabular}{|l|l|}
\hline Modules & Tools and other components \\
\hline Symbolic & Link to analysis and simulation tools (Mathematica) \\
\hline Course Content & $\begin{array}{l}\text { Summaries, lectures, references } \\
\text { General information course objectives, course schedule } \\
\text { Learning involving total immersion, simulation, visualization, } \\
\text { animation } \\
\text { Learning involving audio and video clips }\end{array}$ \\
\hline Assessment & $\begin{array}{l}\text { Grading of true/false, multiple choice, design, analysis } \\
\text { questions } \\
\text { Electronic gradebook }\end{array}$ \\
\hline Course Navigation & Activities schedule, Homework and quizzes \\
\hline Collaborative & Discussion forums, e-mail \\
\hline
\end{tabular}

\section{A. LeProf Implementation}

LeProf was built by integrating locally developed components and third party components in a client-sever architecture. The symbolic, course content, and course navigation modules are implemented on an NCSA web server, the assessment module is implemented in Java on an NT server, and the collaborative module is implemented using the MS FrontPage ${ }^{\mathrm{TM}}$ server extensions. One advantage of this distributed implementation approach is that if one server goes down, students can still access the modules on the other servers. The implementation technology used in each tool or component is listed in Table 11. All modules and tools are linked through HTML in appropriate pages of a course site.

\begin{tabular}{|l|l|}
\hline Tools and other components & Implementation Technology \\
\hline $\begin{array}{l}\text { Link to analysis and simulation tools } \\
\text { (Mathematica) }\end{array}$ & HTML, Mathematica plug-in \\
\hline Summaries, lectures, references & HTML \\
\hline $\begin{array}{l}\text { General information, course objectives, course } \\
\text { schedule }\end{array}$ & HTML \\
\hline $\begin{array}{l}\text { Learning involving total immersion, simulation, } \\
\text { visualization, animation }\end{array}$ & HTML, Java applets \\
\hline $\begin{array}{l}\text { Learning involving audio and video clips } \\
\text { Grading of true/false, multiple choice, design, } \\
\text { analysis questions }\end{array}$ & $\begin{array}{l}\text { HTMa applets, Java Media Framework } \\
\text { (JMF 1.02) }\end{array}$ \\
\hline $\begin{array}{l}\text { Electronic gradebook } \\
\text { Activities schedule }\end{array}$ & $\begin{array}{l}\text { Java applets, Java networking, client- } \\
\text { server }\end{array}$ \\
\hline Homework and quizzes & HTML \\
\hline Discussion forums, e-mail & MS FrontPage \\
\hline
\end{tabular}

Table 11. Implementation Technology Used in LeProf Tools and Components. 
Two important implementation issues taken into account in the implementation of LeProf were scalability and openness. These implementation requirements are met by using the Java language and Java environment. By designing reusable object based components, the system is scaleable. The Java virtual machine is hardware independent thus providing an open framework for running LeProf. Indeed, it has been successfully used in MS Windows as well as Unix environments. Playing audio and video clips is done using the JMF1.0.2 API. The JMF 1.0.2 API models the controls of a VCR or CD player. A Java application or applet using this API can synchronize the simultaneous operation of several Java Media players. The 1.0.2 version of JMF does not transmit (in a streaming fashion) audio nor video over the Internet. It simply allows a client side to playback audio and video which could be pre-fetched from the internet or stored in a local file. The playback is done either using the HTTP or RTP protocols. Table 12 lists all media types currently supported by JMF1.0.2.

\begin{tabular}{|l|l|}
\hline Audio & AIFF, AU, DVI, G.723, GSM, IMA4, MIDI, \\
& MPEG-1 Layer 1/2, PCM, RMF, WAV \\
\hline Video & Apple Graphics (SMC), Apple Animation \\
& (RLE) Cinepak, H.261, H.263, Indeo 3.2, \\
& Motion-JPEG, MPEG-1, Uncompressed \\
\hline File Formats & AVI, QuickTime, Vivo \\
\hline Protocols & File, FTP, HTTP, RTP (RFC 1889/1890) \\
\hline
\end{tabular}

Table 12. Media Types Supported by Java Media Framework (JMF version 1.02).

As noted, LeProf makes extensive use of Java and its object oriented features. Table 13 lists some Java Classes and their Methods in the current version of LeProf. The Element and Circuit classes are used in the course content module whereas the AcceptGrade, UploadGrade, and AccessGrade classes are used in the assessment module. The Element class has methods to draw individual circuit elements (e.g., resistors, voltage or current sources and generic elements). The Circuit class has methods to draw entire circuits, visualize variables in the circuits (e.g., voltage and currents), solve the circuit, check answers entered by students in an interactive fashion, print feedback messages or status and display the values of current variables. The uploadGrade and accessGrade classes are used in client hosts whereas the acceptGrade class is used in the server. Methods sendGrade and readGrade are used by Java Applets to send a grade to the server and read grades from the server respectively. Method writeGrade is used by the server to update students grades accordingly.

\begin{tabular}{|l|l|}
\hline Java Classes (Objects) & Methods (Functions) \\
\hline Element & DrawResistor, drawSource, drawGenElement \\
\hline Circuit & draw, visualize, solve, checkAnswer, printStatus, displayVar \\
\hline AcceptGrade & WriteGrade \\
\hline UploadGrade & SendGrade \\
\hline AccessGrade & ReadGrade \\
\hline
\end{tabular}

\section{B. Comparison with Similar Environments}

Although LeProf supports mainly a symbolically complex learning environment, some features of the other three learning environment are useful for learners with different learning styles. In the following we compare the features of LeProf with other similar virtual environments which primarily support a symbolically complex learning environment. The other environments are: Circuit Tutor, Cyberprof, and Computer-Assisted Personalized Assignment (CAPA). Circuit 
Tutor is a CBT tool for electrical circuits implemented using an early version of ToolBook TM from Asymetrix. Cyberprof is a web based environment developed at the University of Illinois, and CAPA system is a tool for managing assignments developed by Michigan State University. In Table 14, we compare the features of the aforementioned environments with LeProf in terms of all features identified in Table 3.

\begin{tabular}{|c|c|c|c|c|}
\hline Virtual Environment Feature & $\begin{array}{l}\text { Circuit } \\
\text { Tutor }\end{array}$ & Cyberprof & CAPA & LeProf \\
\hline $\begin{array}{l}\text { Lecture notes } \\
\text { Slides, text } \\
\text { Slides, text with audio } \\
\text { Slides, text with audio and video }\end{array}$ & & $\checkmark$ & & \\
\hline Theory readings & & $\checkmark$ & & $\checkmark$ \\
\hline Case studies & & & & \\
\hline Exercises, homework, quizzes & $\sqrt{ }$ & $\checkmark$ & $\checkmark$ & $\sqrt{ }$ \\
\hline Visualization & & & & $\checkmark$ \\
\hline Animation & & & & $\checkmark$ \\
\hline Simulation & & & & $\sqrt{ }$ \\
\hline Peer feedback & & $\sqrt{ }$ & & $\sqrt{ }$ \\
\hline Personalized feedback & $\sqrt{ }$ & $\sqrt{ }$ & $\sqrt{ }$ & $\checkmark$ \\
\hline Feelings are shared & & $\sqrt{ }$ & & $\checkmark$ \\
\hline Apply skill/solve problem activity & & & & $\checkmark$ \\
\hline Teacher is coach/helper & & $\checkmark$ & & $\checkmark$ \\
\hline Teacher is expert/interpreter & & $\sqrt{ }$ & & $\sqrt{ }$ \\
\hline Teacher as task master/guide & & $\checkmark$ & & $\checkmark$ \\
\hline Teacher as model of profession & & & & \\
\hline Expert talk/seminar & & & & $\checkmark$ \\
\hline Self directed (autonomous) learner & & & & $\checkmark$ \\
\hline Learner thinks alone & & & & \\
\hline $\begin{array}{lll}\text { Learner experiences } & \text { being } \\
\text { professional }\end{array}$ & & & & \\
\hline $\begin{array}{l}\text { Learner determines own criteria of } \\
\text { relevance }\end{array}$ & & & & \\
\hline Focus on process & & & & \\
\hline $\begin{array}{l}\text { Information focus on tasks and their } \\
\text { completion }\end{array}$ & $\sqrt{ }$ & $\sqrt{ }$ & $\sqrt{ }$ & $\sqrt{ }$ \\
\hline Symbolic Tool & & & & \\
\hline Information source is here and now & $\sqrt{ }$ & $\checkmark$ & $\checkmark$ & $\sqrt{ }$ \\
\hline Small group discussion & & & & \\
\hline Conferencing & & $\checkmark$ & & $\checkmark$ \\
\hline Synchronous broadcast & & & & \\
\hline Performance judged right or wrong & $\checkmark$ & $\checkmark$ & $\checkmark$ & $\checkmark$ \\
\hline Step by step solution to problem & & & & \\
\hline
\end{tabular}

Table 14. Comparison of Leprof With Other Environments.

\section{A LeProf Site for Electrical Circuits}

The LeProf environment has been used to build a site for a course on electrical circuits for nonmajors. Figure 4 shows a typical Java Applet used by LeProf to provide interactivity. As depicted in Figure 4, the site has been implemented as a typical Web site with a main page that branches to 
many other pages in a hierarchical fashion. As currently configured, it offers the following features: general course information; lesson summaries; detailed examples; learning modules tied to course outline; high interactivity at all levels; extensive use of simulation and visualization; integrated communication system (collaborative module); automatic grading of homework, tests and exams; easy access to grades; random problems for examples and homework; tests.

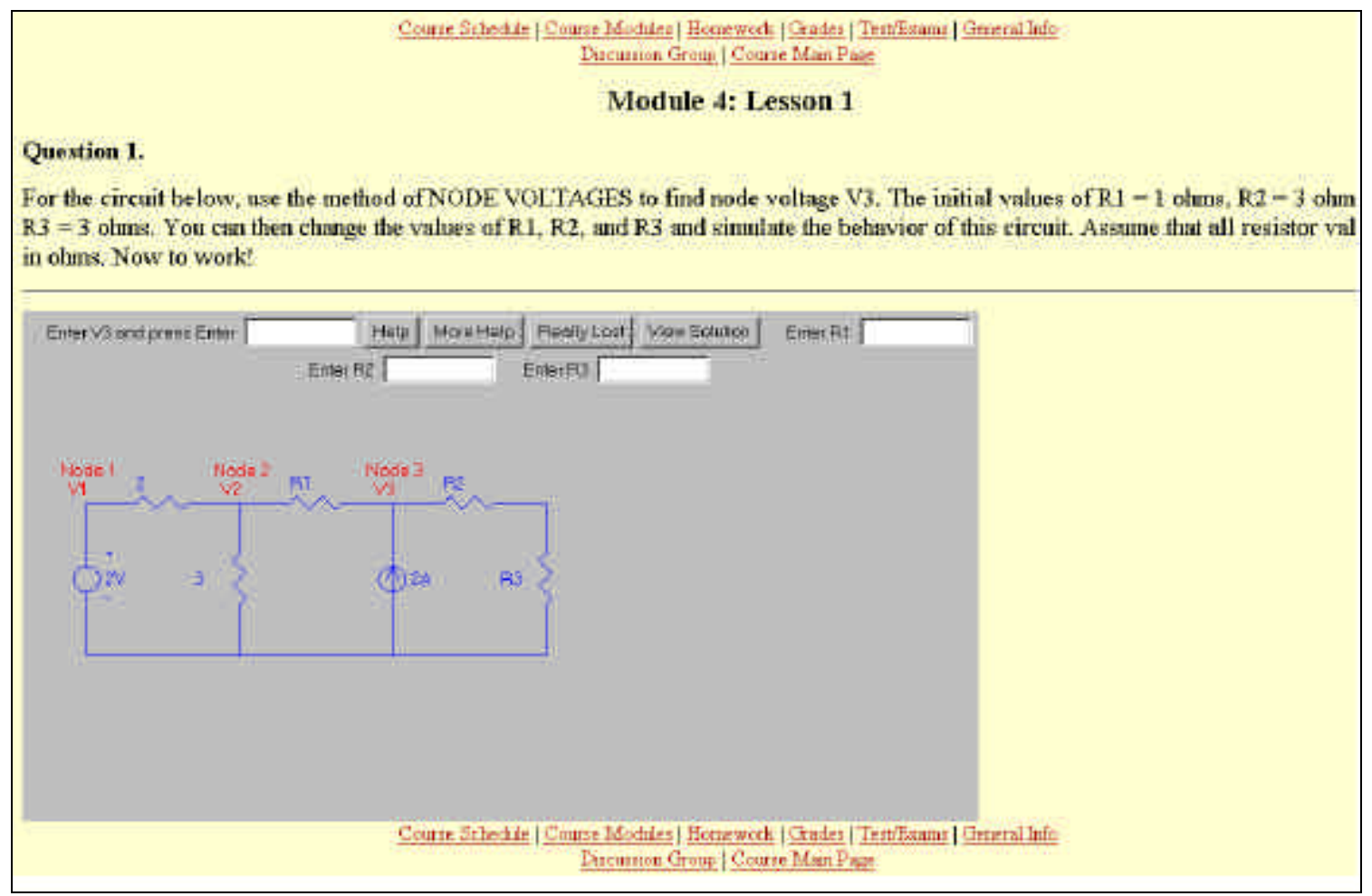

Figure 4. Interactive Virtual Environment for a Course on Electrical Circuits.

Additional details of LeProf can be found in Pimentel [10] or from the site at http://www.kettering.edu/official/acad/ece/ece300/index.html.

The primary means to provide learning experiences in the electrical circuits site is through interactive windows displaying Java applets embedded in web pages. A list of possible experiences for learners includes solving homework problems, working through a learning module, submitting an answer to a problem, obtaining a good (or bad) answer or grade, displaying hints, displaying help messages with varying degree of detail, watching variables being displayed, changing simulation parameters, seeing cause and effect relationships and accessing grades at any time. The actions available to a learner include pressing help buttons, pressing visualization buttons, selecting simulation options, changing simulation parameters, answering questions and submitting answers to problems. Figures 5 and 6 show the feedback obtained when the learner presses the more help and really lost buttons. 


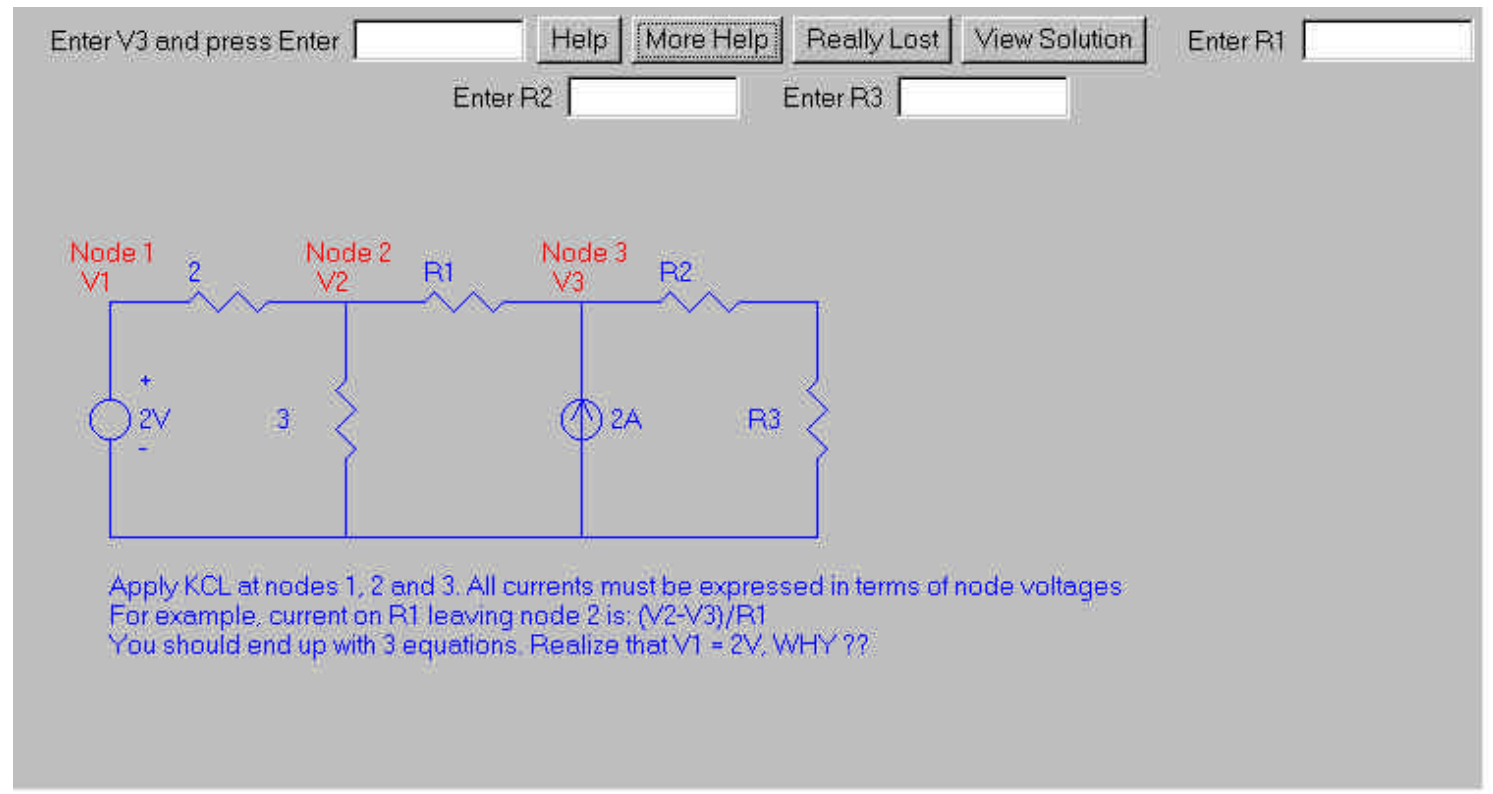

Figure 5. Java Applet Resulting After Pressing the More Help Button.

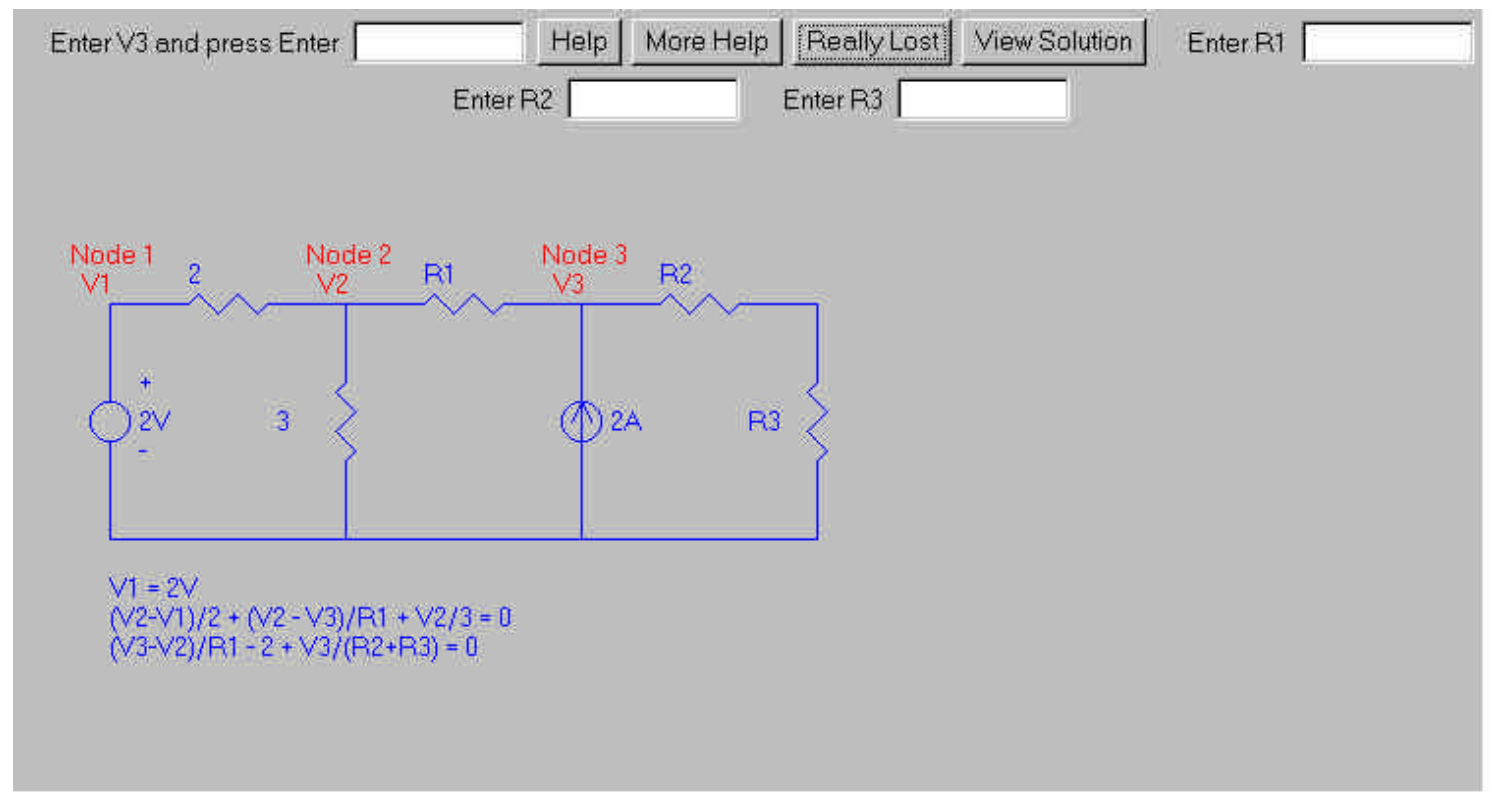

Figure 6. Java Applet Resulting After Pressing the Really Lost Button.

The course modules have options to change some parameters of the circuit and see the effect in the circuit in an immediate fashion thus providing a powerful simulation tool. By using visualization to depict simulation output, learners can more effectively identify relationship between concepts. For example, Figure 7 shows a simulation involving a change in value of resistor R1 from 3 (its initial value) to $30 \mathrm{ohms}$ and the corresponding voltage visualization. Whenever possible, all simulation output in LeProf is communicated to the learner using visualization. 


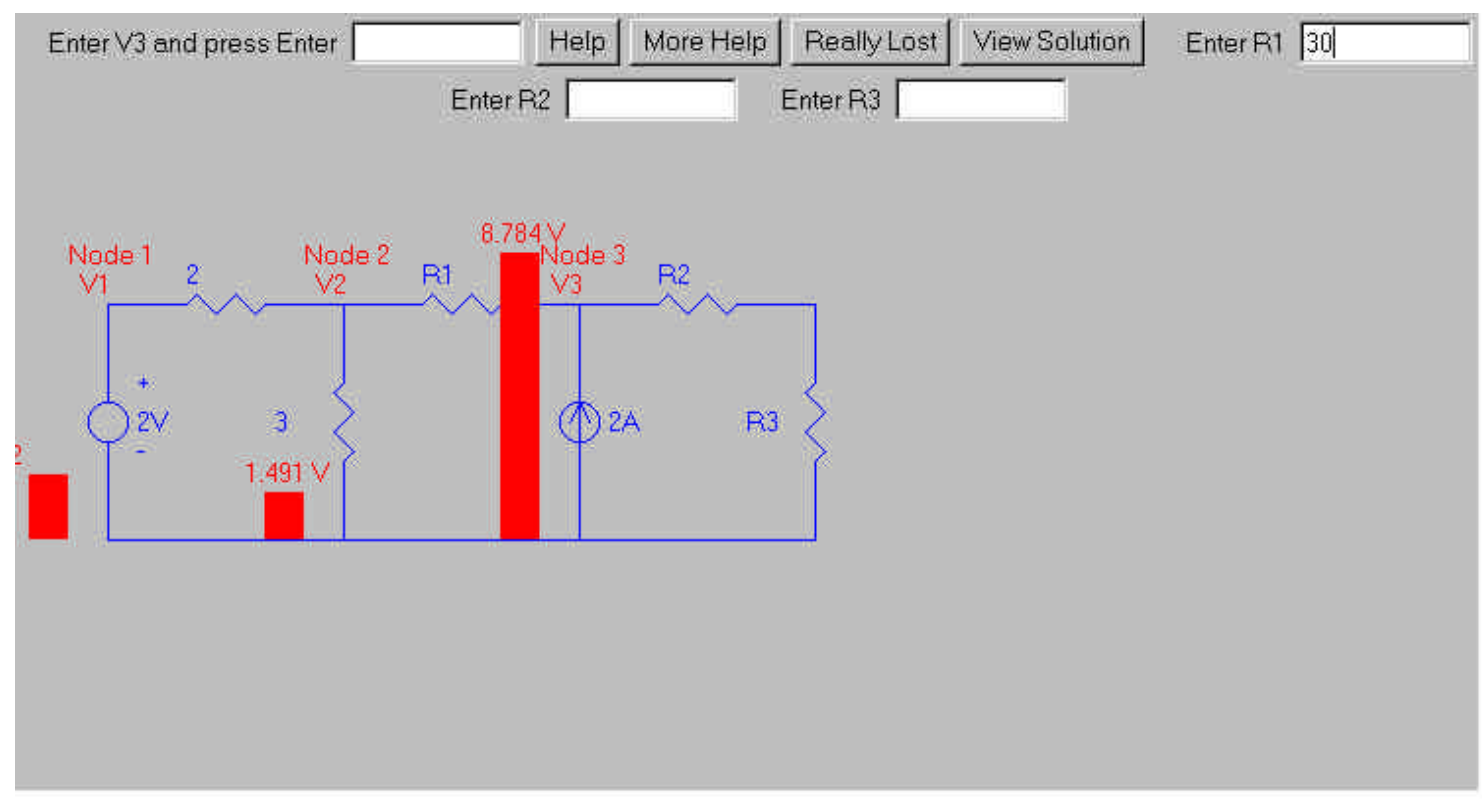

Figure 7. Visualization of Solution After Changing the Value of R1 to 30 Ohms.

The primary perception tool (see Figure 3) is based on visualization and graphical feedback regarding variables, parameters, conditions, hints, answers and help messages. Visualization in electrical circuit education is based on the fact that in a typical circuit problem the most important electrical variables are currents and voltages. Other variables such as energy and power can be easily calculated in terms of these fundamental variables. Thus it is important to have an intuitive way to visualize voltage and currents. Voltage visualization is done using a pressure differential analogy. Current visualization is done using a flow analogy. Associated with any vertical branch in an electrical circuit, the voltage of the element in the branch is depicted as a rectangle whose height is proportional to the actual voltage value as shown in Figure 7. For a horizontal branch, the width of the rectangle is proportional to the voltage. In the case of current visualization, a flow is depicted as rectangles whose width is proportional to the current in the branch.

The assessment module involves the evaluation of learning by the learner rather than from the teacher or instructor. Examples or assessment mechanisms typically used are quizzes and homework. Associated with an evaluation system there is also a performance level that the learner achieves with varying degrees of expectations from the viewpoint of the learner. Although assessment mechanisms are administered by faculty, students use assessment results (e.g., performance) to enhance learning as depicted in Figure 3. LeProf provides an assessment module allowing learners to evaluate their own performance and have an appreciation for understanding content, grades in homework, tests and exams, amount of material covered, speed at which material is worked on and degree of difficulty in handling material. LeProf supports all of this in a timely manner.

\section{Experience with LeProf}

LeProf was used in the course ECE-300 (Electrical Engineering I) during the Winter of 1998 at Kettering University. This course met four times a week and typically students spent one meeting per week working with LeProf under instructor supervision. The main assignment each meeting was to work the course modules and then do the homework problems. Informal and preliminary feedback from students reported that the visualization and simulation did help to understand the subject matter. Although the students were encouraged to use the discussion forum, its use was 
minimal. In general it was found that students were not interested in using any of the tools unless they were getting some credit for it. At this point we do not have enough data for an in-depth evaluation on the effectiveness of LeProf as a learning tool.

\section{CONCLUSIONS}

Experiential learning and the process of learning provide a powerful framework for designing virtual learning environments. By using experiential learning theory we can define learning environments more precisely. We distinguish four types of virtual learning environments that are similar to the four learning environments of Kolb's experiential learning theory. The four virtual learning environments basically define 30 features that are grouped into four fundamental modules and six auxiliary modules. Designing effective virtual environments basically involves identifying appropriate features for a specific subject to construct an educational site while taking into account the various learning styles of potential learners. So far the virtual learning community has recognized the collaborative fundamental module. However, the other three fundamental modules have not been properly recognized or identified as crucial to developing superior virtual learning environments. Two approaches for designing a Net-learning system have been identified: 1) Using an already developed virtual environment and 2) building a virtual environment from its constituent components. Designing and implementing the Process, Symbolic and Guidance fundamental modules are challenging.

The technology based on client-server, Internet, HTML and object oriented systems has proven effective for designing and implementing virtual learning environments. In particular, HTML is well suited for linking all the modules and Java has proven to be a powerful environment for providing features involving a high degree of interaction, visualization, simulation and animation. The Java language was found to be effective because of its straightforward support of graphics, web-interface, client-server systems, multimedia integration and Internet communication. More advances in networking technology are required to successfully design and implement the other modules currently not offered by LeProf (e.g., synchronous broadcast). The advances involve faster communication links and more appropriate protocols to deal with real-time information (e.g., voice and video in synchronous broadcast).

\section{ACKNOWLEDGEMENTS}

Special thanks go to Arlene Hunt and Martin Rosenberg of Kettering University, Anthony DeLellis of Virginia Commonwealth University and Maureen Motter-Hodgson of the University of Calgary for careful reading of an earlier version of this paper and for providing valuable feedback on content and writing. The encouragement of James Gover, Head of the Department of Electrical and Computer Engineering at Kettering, is also acknowledged.

\section{REFERENCES}

1. Bourne, J.R., Net-learning: Strategies for On-Campus and Off-Campus Network-enabled Learning, Journal of Asynchronous Learning Networks (JALN), Vol. 2, Issue 2, Sept. 1998.

2. Kolb, D. A., Experiential Learning, Prentice-Hall, 1984. 
3. Simon, H.A., Why Should Machines Learn?, in Machine Learning: An Artificial Intelligence Approach, R.S. Michalski, J.G. Carbonell, and T.M. Mitchell, Editors, Tioga Pub. Co., pp.25-38, Palo Alto, CA, year?.

4. Famili, A., Integrating Learning and Decision-Making in Intelligent Manufacturing Systems, Intelligent and Robotic Systems, Vol. 3, pp. 117-130, 1990.

5. Fritz, W., Martinez, R.G., Banque, J., Rama, A., Adobbati, R.E. and Sarno, M., The Autonomous Intelligent System, Robotics and Autonomous Systems, Vol 5, pp. 109-125, 1989.

6. Ross, J.M., Instructional Design Paradigms: Is Object-Oriented Design Next? Performance Improvement Quarterly, Vol. 9, No. 3, pp. 23-31, 1996.

7. Chapman, B. L., Enhancing Interactivity and Productivity Through Object Oriented Authoring: An Instructional Designer's Perspective, Journal of Interactive Instruction Development, pp. 3-11, Fall 1994.

8. van der Linden, P., Not Just Java, Prentice-Hall, 1997.

9. Walrand, J., Communication Networks, A First Course, $2^{\text {nd }}$ Ed., WCB/McGraw-Hill, 1998.

10. Wu, C-H. and Irwin, J.D., Emerging Multimedia Computer Communication Technologies, PrenticeHall, 1998.

11. Kuo, F., Effelsberg, W. and Garcia-Luna-Aceves, J.J., Multimedia Communications Protocols and Applications, Prentice-Hall, 1998. 